\title{
Dominant strategy mechanisms with multidimensional types
}

Citation for published version (APA):

Müller, R. J., Gui, H., \& Vohra, R. (2004). Dominant strategy mechanisms with multidimensional types. METEOR, Maastricht University School of Business and Economics. METEOR Research Memorandum No. 046 https://doi.org/10.26481/umamet.2004046

Document status and date:

Published: 01/01/2004

DOI:

10.26481/umamet.2004046

Document Version:

Publisher's PDF, also known as Version of record

\section{Please check the document version of this publication:}

- A submitted manuscript is the version of the article upon submission and before peer-review. There can be important differences between the submitted version and the official published version of record.

People interested in the research are advised to contact the author for the final version of the publication, or visit the DOI to the publisher's website.

- The final author version and the galley proof are versions of the publication after peer review.

- The final published version features the final layout of the paper including the volume, issue and page numbers.

Link to publication

\footnotetext{
General rights rights.

- You may freely distribute the URL identifying the publication in the public portal. please follow below link for the End User Agreement:

www.umlib.nl/taverne-license

Take down policy

If you believe that this document breaches copyright please contact us at:

repository@maastrichtuniversity.nl

providing details and we will investigate your claim.
}

Copyright and moral rights for the publications made accessible in the public portal are retained by the authors and/or other copyright owners and it is a condition of accessing publications that users recognise and abide by the legal requirements associated with these

- Users may download and print one copy of any publication from the public portal for the purpose of private study or research.

- You may not further distribute the material or use it for any profit-making activity or commercial gain

If the publication is distributed under the terms of Article $25 \mathrm{fa}$ of the Dutch Copyright Act, indicated by the "Taverne" license above, 


\title{
Dominant Strategy Mechanisms with Multidimensional Types
}

\author{
Hongwei Gui* Rudolf Müller ${ }^{\dagger} \quad$ Rakesh V. Vohra ${ }^{\ddagger}$ \\ October 18, 2004
}

\begin{abstract}
This paper provides a characterization of dominant strategy mechanisms with quasi-linear utilities and multi-dimensional types for a variety of preference domains. These characterizations are in terms of a monotonicity property on the underlying allocation rule.
\end{abstract}

Keywords: Dominant Strategy, Farkas Lemma, Combinatorial Auctions.

${ }^{*}$ Department of Managerial Economics and Decision Sciences, Kellogg Graduate School of Management, Northwestern University, Evanston IL 60208.

${ }^{\dagger}$ Department of Quantitative Economics, Faculty of Economics and Business Administration, Maastricht University, 6200 MD Maastricht, The Netherlands

${ }^{\ddagger}$ Department of Managerial Economics and Decision Sciences, Kellogg Graduate School of Management, Northwestern University, Evanston IL 60208. 


\section{Introduction}

In this paper we show how to characterize dominant strategy mechanisms in environments with quasi-linear utilities and multi-dimensional types. While the characertizations themselves are of interest, we believe the techniques are of independent interest and will be of use in other applications.

We consider direct revelation mechanisms where types are private. With a change in notation the results would extend to the interdependent value setting and ex-post incentive compatibility.

Any direct mechanism can be decomposed into two parts: an allocation rule and a payment rule. The allocation rule determines the allocation of resources as a function of the profile of reported types. The payment rule determines the payment each agent must make as a function of the profile of reported types. Recent characterizations of dominant strategy mechanisms (eg. Jehiel and Moldovanu (2001), Krishna and Perry (1997)) are in terms of the utility that will be delivered to the agents. Here we offer characterizations in terms of a monotonicity property on the allocation rule itself. Examples of characterizations with this flavor that appear in the literature are Myerson (1981), Bikhchandani, Chatterji and Sen (2003) and Lavi, Mu'alem and Nisan (2003). In fact the characterizations offered in those papers can all be derived (as we illustrate) using the technique reported here. We also derive a characterization for a preference domain not covered by previous work. Specifically, the methods of Lavi, Mu'alem and Nisan do not apply when the type space is compact. The arguments of Bikhchandani, Chatterji and Sen ( 2003) apply to one specific environment, i.e, additive marginal valuations.

To give a flavor of the results that are obtained, we describe the result of Bikhchandani, Chatterji and Sen (2003) that inspired this paper. That paper considers the allocation of $k$ indivisble units of the same good to agents with additive marginal valuations. Specifically, each agent has a type $t \in[0,1]^{k}$. Their value for the $i^{t h}$ unit is $t_{i}$. Thus an agent with type $t$ who receives $\alpha$ units of the good derives a utility of $\sum_{i=1}^{\alpha} t_{i}$.

Fix an agent $i$ and hold the profile of types, $\mathbf{t}^{-i}$, of the other agents fixed. Suppose the allocation rule, $f$ will assign a quantity $f\left(t, \mathbf{t}^{-i}\right)$ to agent $i$ if she reports type $t$. Bikhchandani, Chatterji and Sen show that the rule $f$ is 
dominant strategy incentive compatible iff.

$$
\sum_{j=1}^{f\left(t, \mathbf{t}^{-i}\right)} t_{j}-\sum_{j=1}^{f\left(s, \mathbf{t}^{-i}\right)} t_{j} \geq-\left[\sum_{j=1}^{f\left(s, \mathbf{t}^{-i}\right)} s_{j}-\sum_{j=1}^{f\left(t, \mathbf{t}^{-i}\right)} s_{j}\right] \forall s, t .
$$

In particular, if $f\left(t, \mathbf{t}^{-i}\right)>f\left(s, \mathbf{t}^{-i}\right)$ then $\sum_{j=f\left(s, \mathbf{t}^{-i}\right)+1}^{f\left(t, \mathbf{t}^{-i}\right)} t_{j} \geq \sum_{j=f\left(s, \mathbf{t}^{-i}\right)+1}^{f\left(t, \mathbf{t}^{-i}\right)} s_{j}$. Bikhchandani, Chatterji and Sen call this condition nondecreasing in marginal utility. We show that a generalization of this condition characterizes dominant strategy mechanisms in other environments.

The next section introduces notation. The subsequent section introduces the condition and a description of an approach to characterizing dominant strategy mechanisms using this condition. We then give four applications of the approach.

\section{Notation}

$T$ is a set of (multi-dimensional) types, $T \subseteq \Re^{k}$. Denote by $T^{n}$ the set of all $n$-agent profiles of types ${ }^{1}$. An element of $T^{n}$ will be usually be written as $\left(t^{1}, t^{2}, \ldots, t^{n}\right)$ or $\mathbf{t}$.

$\Gamma$ is a finite set of at least three outcomes. An allocation rule is a function

$$
f: T^{n} \rightarrow \Gamma .
$$

We assume that $f$ is onto, i.e., for each $\alpha \in \Gamma$ there is a $\mathbf{t} \in T$ such that $f(\mathbf{t})=\alpha$. A payment rule is a function

$$
P: T^{n} \rightarrow \Re^{n},
$$

that is, if the reported profile is $\left(t^{1}, \ldots, t^{n}\right)$ agent $i$ makes a payment of $P_{i}\left(t^{1}, \ldots, t^{n}\right)$. Utilities are quasi-linear. The value that agent $i$ with type $t \in T$ assigns to an allocation $\alpha \in \Gamma$ is denoted $v^{i}(\alpha \mid t)$. Let $R_{\alpha}=\left\{\mathbf{t} \in T^{n}\right.$ : $f(\mathbf{t})=\alpha\} \forall \alpha \in \Gamma$. Clearly $T^{n}=\cup_{\alpha \in \Gamma} R_{\alpha}$.

Beginning in section 3.3 we will assume that $v^{i}(\alpha \mid t)$ is linear in $t$. Therefore, we can identify each $\alpha \in \Gamma$ with a vector $a^{\alpha} \in \Re^{k}$ such that $v^{i}(\alpha \mid t)=$

\footnotetext{
${ }^{1}$ The type space need not be identical across agents. We make the assumption for simplicity of notation
} 
$a^{\alpha} \cdot t$. Note that this vector may depend on the identity of the agent, but in most cases will be identical for all agents.

Let us describe three cases.

1. Homogenous, multi-item auctions with additive valuations. Suppose we have $k$ units of a homogenous good to allocate. The type of an agent is a vector in $\Re_{+}^{k}$ whose $j^{\text {th }}$ component is the marginal value for the $j^{\text {th }}$ unit. Each $\alpha \in \Gamma$ can be represented by an integral vector in $\Re_{+}^{k}$ whose $i^{\text {th }}$ component represents the quantity allocated to agent $i$ and sum of components is $k$. The $i^{\text {th }}$ component will be denoted $\alpha_{i}$, and $v^{i}(\alpha \mid t)=\sum_{j=1}^{\alpha_{i}} t_{j}$. Alternatively, for agent $i$ we can represent all $\alpha$ with same $\alpha_{i}$ by a vector $a^{\alpha} \in \Re_{+}^{k}$ with $\alpha_{i}$ leading 1 's, and the rest of its coefficients being equal to 0 , in order to have a representation such that $v^{i}(\alpha \mid t)=a^{\alpha} \cdot t$.

2. Combinatorial Auctions. We have a set $M$ of distinct goods to allocate. The type of an agent is a vector in $\Re_{+}^{2^{|M|}}$ with one component for each subset of $M$ that corresponds to the value assigned to that subset. If the allocation $\alpha$ assigns the set $S \subseteq M$ to agent $i$ with type $t$ then $v^{i}(\alpha \mid t)=t_{S}$. The vector representation of $\alpha$ is now given by the unit vectors in $\Re^{2^{|M|}}$.

3. Unrestricted Preferences. Following the previous example, but now we want to allow for the possibility that an agents payoff depends not just on the goods assigned to him, but the goods assigned to other agents as well. In this case a type would be a vector with one component for each allocation, i.e. in $\Re_{+}^{|\Gamma|}$.

We can fold the previous two models into the set up of unrestricted preferences. Consider the combinatorial auction example. An agent would be indifferent between any two allocations that give him the same set. Thus the set of types of an agent would not 'fill out' $\Re_{+}^{|\Gamma|}$, i.e., they form a subspace of $\Re_{+}^{|\Gamma|}$. This absence of full dimensionality is the reason why results obtained for unrestricted preferences will not automatically apply to more restrictive settings. 
An allocation rule $f$ is dominant strategy incentive compatible if there exists a payment rule $P$ such that for all agents $i$ and all types $s \neq t$ :

$$
v^{i}\left(f\left(t, \mathbf{t}^{-i}\right) \mid t\right)-P_{i}\left(t, \mathbf{t}^{-i}\right) \geq v^{i}\left(f\left(s, \mathbf{t}^{-i}\right) \mid t\right)-P_{i}\left(s, \mathbf{t}^{-i}\right) \forall \mathbf{t}^{-i} .
$$

Our goal is to characterize the class of dominant strategy allocation rules.

In the sequel we fix an agent $i$ and a profile of types for the other $n-1$ agents. For this reason we suppress dependence on the index $i$ and $t^{-i}$ in the sequel unless we say otherwise.

If we restrict our attention to agent $i$, we will always identify allocations between which agent $i$ is indifferent. By this we can assume that for all $\alpha \neq \beta$ of $\Gamma$ there exists a type $t$ such that $v(\alpha \mid t) \neq v(\beta \mid t)$. Furthermore, we will for fixed $t^{-i}$ restrict to $\Gamma=\left\{\alpha \mid \exists t \in T\right.$ such that $\left.f\left(t, t^{-i}\right)=\alpha\right\}$. Accordingly, from now on $R_{\alpha}=\left\{t \mid f\left(t, t^{-i}\right)=\alpha\right\}$. Under this convention inequality (1) becomes

$$
v(f(t) \mid t)-P(t) \geq v(f(s) \mid t)-P(s) .
$$

\subsection{2 -cycle constraint}

Reversing the roles of $t$ and $s$ in (2) implies

$$
v(f(s) \mid s)-P(s) \geq v(f(t) \mid s)-P(t) .
$$

Adding (2) to (3) yields

$$
v(f(t) \mid t)+v(f(s) \mid s) \geq v(f(s) \mid t)+v(f(t) \mid s) .
$$

Rewriting:

$$
v(f(t) \mid t)-v(f(s) \mid t) \geq-[v(f(s) \mid s)-v(f(t) \mid s)] .
$$

We call (4) a 2-cycle inequality. That the 2-cycle inequality holds for every pair $s, t \in T$ is a necessary condition of dominant strategy incentive compatibility. We show that this is a sufficient condition for a variety of preference domains. ${ }^{2}$ To summarize, our theorems are of the following form: an allocation rule is dominant strategy incentive compatible iff. it satisfies

\footnotetext{
${ }^{2}$ The assumption that $|\Gamma| \geq 3$ is what rescues this statement from being trivial.
} 
the 2-cycle inequality. The non-decreasing in marginal utility condition of Bikhchandani, Chatterji and Sen is an instance of the 2-cycle inequality. The characterization results for other domains in Lavi, Mu'alem and Nisan (2003) are in terms of 2-cycle inequalities as well. ${ }^{3}$

\section{The approach}

Our approach is based on the fact that the constraints (1) have a natural network interpretation. To see this, it will be useful to rewrite inequality (2) as

$$
P(t)-P(s) \leq v(f(t) \mid t)-v(f(s) \mid t) .
$$

As is explained below, such a system is a linear programming dual to the problem of finding a shortest path in an appropriate network. The reader familiar with the theory of network flows can skip the next subsection.

\subsection{An aside on paths and cycles}

Let $N$ be a finite set of indices and $E$ the set of all ordered pairs of elements of $N$. Associated with each pair $(i, j) \in E$ is a number $w_{i j}$. Consider the following inequality system:

$$
x_{j}-x_{i} \leq w_{i j} \forall(i, j) \in E .
$$

We can associate a network with (6) in the following way. Each element of $N$ is interpreted as vertex, and to each ordered pair $(i, j) \in E$ we associate a directed edge from vertex $i$ to vertex $j$. Each edge $(i, j) \in E$ is assigned a length of $w_{i j}$.

A standard result (which follows from the Farkas lemma) is that (6) is feasible iff. the associated network contains no negative length cycle. ${ }^{4}$ Second, if the system is feasible, one solution is to set each $x_{j}$ equal to the length of the shortest path from an arbitrarily chosen root vertex.

\footnotetext{
${ }^{3}$ Lavi, Mu'alem and Nisan use the term weak monotonicity instead of 2-cycle inequality.

${ }^{4}$ If the direction of the inequality in (6) is reversed, then the system is feasible iff. the network has no positive length cycle.
} 
To see that cycles should play a role, consider the pair of inequalities $x_{j}-x_{i} \leq w_{i j}$ and $x_{i}-x_{j} \leq w_{j i}$. Feasibility requires that $w_{i j} \geq x_{j}-x_{i} \geq-w_{j i}$, i.e. $w_{i j}+w_{j i} \geq 0$. In words the length of the 2 -cycle $i \rightarrow j \rightarrow i$ is nonnegative. Similarly, cycles with more than 2 vertices must not be negative.

Establishing our characterization of dominant strategy incentive compatible mechanisms amounts to proving that an associated network contains no negative length cycles when all its 2-cyles have non-negative length. Nonnegativity of all 2-cycles does not in general imply non-negativity of all cycles.

\subsection{Allocation graph}

Given the observations above, a natural step is to associate a vertex with each type and a directed edge between each ordered pair of vertices. Define the length of the edge directed from type $s$ to type $t$ by

$$
l(s, t)=v(f(t) \mid t)-v(f(s) \mid t)
$$

and call this graph $T_{f}$. For technical reasons we allow for loops. But observe that $l(t, t)=0$.

Rochet (1987) was the first to observe the relation between negative cycles and dominant strategy incentive compatibility. It was rederived again in Rozenshtrom (1999).

Theorem 1 (Rochet(1987)) Let $T$ be any type space, $n \geq 2$ be the number of agents with quasi-linear utilities over a set $\Gamma$ of outcomes and $f: T^{n} \rightarrow \Gamma$ an allocation rule. The following statements are equivalent:

(1) $f$ is dominant strategy incentive compatible.

(2) For every agent $i$, for every report $\mathbf{t}^{-i}$, the corresponding graph $T_{f}$ does not have a finite cycle of negative length.

\section{Proof}

(as in Rochet (1987)) (1) $\Rightarrow(2)$

Let $t_{0} \in T$ be an arbitrary, but fixed type. Define

$$
P(t)=\inf \left(\sum_{i=0}^{k} l\left(t_{i}, t_{i+1}\right) \mid k \geq 0, t_{1}, \ldots, t_{k+1} \in T, t_{k+1}=t\right) .
$$


Observe that $P\left(t_{0}\right)=0$. Observe also that $P(t)$ is larger than or equal to $-l\left(t, t_{0}\right)$, and thus larger than $-\infty$, because if not then $\Gamma_{T}$ would have a negative cycle. Finally for every $t, t^{\prime} \in T$ :

$$
P\left(t^{\prime}\right) \leq P(t)+l\left(t, t^{\prime}\right)=P(t)+v\left(f\left(t^{\prime}\right) \mid t^{\prime}\right)-v(f(t) \mid t) .
$$

$(2) \Rightarrow(1)$

Let $t_{1}, \ldots, t_{k}, t_{k+1}=t_{1}$ be a finite cycle. Since $f$ is dominant strategy incentive compatible there exists a payment function $P$ such that:

$$
\sum_{i=1}^{k} l\left(t_{i}, t_{i+1}\right)=\sum_{i=1}^{k} v\left(f\left(t_{i+1} \mid t_{i+1}\right)-v\left(f\left(t_{i}\right) \mid t_{i+1}\right) \geq \sum_{i=1}^{k} P\left(t_{i+1}\right)-P\left(t_{i}\right)=0 .\right.
$$

Observe that for any $t, t^{\prime} \in T$ such that $f(t)=f\left(t^{\prime}\right)$, it must be that $l\left(t, t^{\prime}\right)=0$. It is easy to see that in this case $P(t)=P\left(t^{\prime}\right)$.

The well known 'monotonicity' characterization of dominant strategy allocation rules when types are one dimensional follows from this cycle characterization.

We find it more convenient to work with a different but related graph, called the allocation graph. We associate with each element $\alpha \in \Gamma$ a vertex. The length, $l(\alpha, \beta)$ of an edge directed from allocation $\alpha$ to allocation $\beta$ is given by by

$$
l(\alpha, \beta)=\inf _{s \in R_{\beta}}[v(\beta \mid s)-v(\alpha \mid s)] .
$$

Symmetrically, we associate an edge directed from $\beta$ to $\alpha$ with length:

$$
l(\beta, \alpha)=\inf _{t \in R_{\alpha}}[v(\alpha \mid t)-v(\beta \mid t)] .
$$

Denote the graph by $\Gamma_{f}$. Notice that if the 2-cycle inequality holds,

$$
l(\alpha, \beta)+l(\beta, \alpha) \geq 0 \forall \alpha, \beta \in \Gamma .
$$

From Rochet's theorem (or direct appeal to known results) we obtain the following. 
Corollary 1 Let $T$ be any type space, $n \geq 2$ be the number of agents with quasi-linear utilities over a set $\Gamma$ of outcomes and $. f: T^{n} \rightarrow \Gamma$ an allocation rule. The following statements are equivalent:

(1) $f$ is dominant strategy incentive compatible.

(2) For every agent, for every report $\mathbf{t}^{-i}$, the corresponding graph $\Gamma_{f}$ does not have a finite cycle of negative length.

We show in the following that for certain environments $\Gamma_{f}$ does not have a negative cycle iff. it does not have a negative 2-cycle. We assume from now on that $v(\alpha \mid$.) is linear in $t$ for all $\alpha \in \Gamma$.

\subsection{Describing $R_{\alpha}$}

In this section we show that if (7) holds the set $R_{\alpha}$ is contained in a polyhedron $Q_{\alpha}$ for all $\alpha \in \Gamma$, and these polyhedra can be chosen such that the intersection of $T$ with the interior, $I\left(Q_{\alpha}\right)$, of $Q_{\alpha}$ is contained in $R_{\alpha}$. Observe that for $t \in R_{\alpha}$ and $s \in R_{\beta}$ we have

$$
v(\alpha \mid t)-v(\beta \mid t) \geq-[v(\beta \mid s)-v(\alpha \mid s)] .
$$

Therefore

$$
v(\beta \mid t)-v(\alpha \mid t) \leq \inf _{s \in R_{\beta}}[v(\beta \mid s)-v(\alpha \mid s)]=l(\alpha, \beta) .
$$

Thus $R_{\alpha}$ is a subset of

$$
Q_{\alpha}=\left\{x \in \Re^{k}: v(\beta \mid x)-v(\alpha \mid x) \leq l(\alpha, \beta) \forall \beta \neq \alpha\right\} .
$$

This is a polyhedron because we assume that $v$ is linear in $x$.

Now assume $I\left(Q_{\alpha}\right) \neq \emptyset$ and consider a $t$ of $T \cap I\left(Q_{\alpha}\right)$. We show that $t \in R_{\alpha}$. Observe that for all $\beta \neq \alpha, t \notin R_{\beta}$. Indeed, otherwise we get the contradiction $^{5}$

$$
v(\beta \mid t)-v(\alpha \mid t)<l(\alpha, \beta)<v(\beta \mid t)-v(\alpha \mid t) .
$$

\footnotetext{
${ }^{5}$ We make use of $v(\alpha \mid.) \neq v(\beta \mid$.$) for \alpha \neq \beta$. This ensures that inequality (8) has non-zero left-hand-side, and thus every point in the interior of $Q_{\alpha}$ satisfies (8) with strict inequality.
} 
Notice that there is a one-to-one correspondence between the constraints of these polyhedra and edges of $\Gamma_{f}$. Specifically, the constraint $v(\beta \mid x)-$ $v(\alpha \mid x) \leq l(\alpha, \beta)$ corresponds to the edge $(\alpha, \beta)$.

\subsection{Implications of negative cycles in $\Gamma_{f}$}

Corrollary 1 says that $f$ is dominant strategy incentive compatible iff. $\Gamma_{f}$ does not contain a negative cycle. We show below that in many cases, $\Gamma_{f}$ does not contain a negative cycle iff. $\Gamma_{f}$ does not contain a negative 2-cycle. In other words, the 2-cycle inequality is a necessary and sufficient condition for an allocation rule to be dominant strategy incentive compatible.

Necessity, as we have seen already is easy to establish. We focus on sufficiency. Here is a broad outline of the argument.

Suppose $\Gamma_{f}$ has no negative 2-cycle. Assume, for a contradiction, that $\Gamma_{f}$ has a negative cycle. From Section 3.3 we deduce that the type space $T$ can be covered by $\bigcup_{\alpha \in \Gamma} Q_{\alpha}$. Invoking the existence of the negative cycle and the Farkas lemma enables us to construct a vector $x \notin \cup_{\alpha \in \Gamma} Q_{\alpha}$, and thus $x \notin \cup_{\alpha \in \Gamma} R_{\alpha}$. With some additional effort we show that $x$ can be chosen so that $x \in T$. This contradicts the fact that $T \subseteq \cup_{\alpha \in \Gamma} R_{\alpha}$.

In the remainder of this section we describe how to derive the existence of an $x \notin \cup_{\alpha \in \Gamma} R_{\alpha}$. Establishing that $x \in T$ is discussed for each environment separately.

Suppose $\Gamma_{f}$ has a negative cycle, i.e. there exists a sequence of vertices $\alpha_{1}, \alpha_{2}, \ldots, \alpha_{r}, \alpha_{r+1}=\alpha_{1}$ such that

$$
l\left(\alpha_{1}, \alpha_{2}\right)+l\left(\alpha_{2}, \alpha_{3}\right)+\ldots+l\left(\alpha_{r}, \alpha_{1}\right)<0 .
$$

Consider the following system:

$$
v\left(\alpha_{j+1} \mid x\right)-v\left(\alpha_{j} \mid x\right) \geq l\left(\alpha_{j}, \alpha_{j+1}\right)+\epsilon \forall j=1, \ldots, r .
$$

Basically we take one inequality from each $Q_{\alpha_{j}}$ for $j=1, \ldots, r$ and reverse it. Here $\epsilon>0$ is chosen to be sufficiently small such that the length of the cycle, even after augmenting the length of edges by $\epsilon$, is still negative.

Feasibility of the system (9) implies the existence of a vector $x$ such that $x \notin \cup_{j=1}^{r} Q_{\alpha_{j}}$. Add to this system one (reversed) inequality from each $Q_{\alpha}$ for 
$\alpha \in \Gamma \backslash\left\{\alpha_{1}, \ldots, \alpha_{r}\right\}$. We do so in such a way that the corresponding edges chosen contain only one cycle, the one on $\alpha_{1}, \ldots, \alpha_{r}$. One way of doing this is to choose for every vertex not on the cycle an edge directed into the cycle. Let $E$ denote the subset of edges chosen, and consider the system:

$$
v(\beta \mid x)-v(\alpha \mid x) \geq l(\alpha, \beta)+\epsilon \forall(\alpha, \beta) \in E .
$$

If (10) is feasible it means there is an $x \in \Re^{k}$ such that $x \notin \cup_{\alpha \in \Gamma} Q_{\alpha}$, and thus $x \notin \cup_{\alpha \in \Gamma} R_{\alpha}$. If in addition $x \in T$, we would have identified a type not in $\cup_{\alpha \in \Gamma} R_{\alpha}$, a contradiction to $\cup_{\alpha \in \Gamma} R_{\alpha}=T$.

The difficulties arise in trying to prove that (10) has a feasible solution contained in $T$. A substitution $Y_{\alpha}=v(\alpha \mid x)$ will reveal some structure that can be exploited. Linearity of $v$ implies there is a matrix $A$ such that $Y=A x$.

(10) becomes:

$$
\begin{gathered}
Y_{\beta}-Y_{\alpha} \geq l(\alpha, \beta)+\epsilon \forall(\alpha, \beta) \in E \\
Y=A t \text { for some } t \in T .
\end{gathered}
$$

Focus on the first set of constraints. The graph $(\Gamma, E)$ does not contain a positive length cycle. So the first set of constraints are feasible. Indeed choose an arbitrary node $\alpha_{0} \in \Gamma$ and compute and set $Y_{\alpha}$ equal to length of a longest path from $\alpha_{0}$ to $\alpha$. The challenge will be to show that amongst the set of feasible solutions to the first set of constraints is one, $Y$, say, that also satisfies $Y=A t$ for some $t \in T$.

\section{Unrestricted quasi-linear preferences}

Here an agents type is a vector from $\Re^{|\Gamma|}, \Re_{+}^{|\Gamma|}$ or $[0,1]^{|\Gamma|}$. The $\alpha \in \Gamma$ component of $t$, denoted $t_{\alpha}$ is the value that the agent with type $t$ attaches to allocation $\alpha$.

Theorem 2 Suppose preferences are unrestricted and the type space $T$ is $\Re^{|\Gamma|}, \Re_{+}^{|\Gamma|}$ or $[0,1]^{|\Gamma|}$. An allocation rule $f$ is dominant strategy incentive compatible iff.

$$
t_{f(t)}-t_{f(s)} \geq-\left[s_{f(s)}-s_{f(t)}\right] \forall s, t \in T \text {. }
$$




\section{Proof}

Suppose first $T=\Re^{|\Gamma|}$. We confine ourselves to a proof of sufficiency. The system (10) in this case reduces to:

$$
x_{\beta}-x_{\alpha} \geq l(\alpha, \beta)+\epsilon \forall(\alpha, \beta) \in E .
$$

Notice that (11) is feasible and that any solution belongs to $T$.

Now suppose that $T=[0,1]^{|\Gamma|}$. Before proceeding it is useful to see where the previous argument fails as this suggests a natural fix. In this case $|l(\alpha, \beta)| \leq 1$ for all $(\alpha, \beta)$. An arbitrary solution $x$ of (11) may not reside in $[0,1]^{|\Gamma|}$. This may happen in particular when $l(\alpha, \beta)=1$ for some $(\alpha, \beta) \in E$. In which case $x_{\beta}-x_{\alpha} \geq 1+\epsilon$ requiring that either $x_{\alpha}>1$ or $x_{\beta}<0$. We can modify (11) to account for this difficulty by conisdering the following system:

$$
\begin{aligned}
x_{\beta}-x_{\alpha} & \geq \min \{l(\alpha, \beta)+\epsilon, 1\} \forall(\alpha, \beta) \in E(S 1) \\
x_{\gamma} & \in[0,1] \forall \gamma \in \Gamma
\end{aligned}
$$

Considering again the pair $(\alpha, \beta)$ such that $l(\alpha, \beta)=1$, (S1) yields

$$
x_{\beta}-x_{\alpha} \geq 1 \text {. }
$$

If $l(\alpha, \beta)=1>-l(\beta, \alpha)$ this inequality is still sharp enough to allow us to exclude $x$ from $R_{\alpha}$. Thus another difficulty arises when $l(\alpha, \beta)=-l(\beta, \alpha)=$ 1. This second difficulty motivates a definition. Call a pair $\{\alpha, \beta\}$ tight if $l(\alpha, \beta)=-l(\beta, \alpha)=1$. We assume first that $\Gamma_{f}$ has no tight pairs.

Suppose for a contradiction that $\Gamma_{f}$ has a negative length cycle. Amongst all such cycles, choose one, $C$, say, that has as few vertices as possible. Let $C=\left\{\alpha_{1}, \ldots, \alpha_{r}\right\}$ and $d\left(\alpha_{j}, \alpha_{k}\right)$ be the length of the path on $C$ going from $\alpha_{j}$ to $\alpha_{k}$ with respect to edge lengths $l\left(\alpha_{i}, \alpha_{i+1}\right)$. If $d\left(\alpha_{j}, \alpha_{k}\right) \geq 1$, we can replace this path by the edge $\left(\alpha_{j}, \alpha_{k}\right)$, producing a negative cycle, with fewer vertices. Thus $d\left(\alpha_{j}, \alpha_{k}\right)<1$ for all $j, k$. We use this property to show that there is a solution to

$$
\begin{aligned}
x_{\beta}-x_{\alpha} & \geq \min \{l(\alpha, \beta)+\epsilon, 1\} \forall(\alpha, \beta) \in C \\
x_{\gamma} & \in[0,1] \forall \gamma \in C
\end{aligned}
$$


Consider the following linear program.

$$
\begin{aligned}
\min y-z & (S 3) \\
\text { s.t. } x_{\beta}-x_{\alpha} & \geq \min \{l(\alpha, \beta)+\epsilon, 1\} \forall(\alpha, \beta) \in C \\
x_{\gamma}-z & \geq 0 \forall \gamma \in C \\
y-x_{\gamma} & \geq-1 \forall \gamma \in C
\end{aligned}
$$

This is the dual to the problem of finding a longest path in a network that contains the cycle $C$ plus two additional vertices. One vertex is associated with the variable $z$, call it the source and the other is associated with $y$ called the sink. For each $\alpha \in C$ there is an edge directed from the source into $\alpha$ of length 0 . From each $\alpha \in C$ there is an edge directed into the sink of length -1 . It is easy to see that this network has no positive length cycle and so a longest path from source to sink exists. Notice also that any path must start at the source, enter some vertex $\alpha_{i} \in C$, traverse $C$ and leave for the sink via some vertex $\alpha_{k} \in C$. The length of this path is at most $d\left(\alpha_{i}, \alpha_{k}\right)+(k-i) \epsilon-1 \leq 0$. Since the variables in (S3) correspond to longest path lengths from the source, there is an optimal solution $\left(x^{*}, z^{*}, y^{*}\right)$ to (S3) where $z^{*}=0$ and $y^{*}<0$. Hence $x_{\gamma}^{*} \geq 0$ for all $\gamma \in \Gamma$. Also $-1 \leq y^{*}-x_{\gamma}^{*} \leq-x_{\gamma}^{*}$, i.e. $x_{\gamma}^{*} \leq 1$ for all $\gamma \in C$. Therefore $x^{*}$ is a feasible solution to $(\mathrm{S} 2)$.

If $x_{\alpha}^{*}<1$ for all $\alpha \in C$ we can construct a new solution $x$ to (S2) where $x_{\alpha}=1$ for at least one $\alpha \in C$. Simply increase all $x_{\alpha}^{*}$ at the same rate until one of them becomes 1. All constraints of (S2) are satisfied. For all $\gamma \notin C$ set $x_{\gamma}=0$. It is easy to see that $x \in[0,1]^{|\Gamma|}$. We claim that $x \notin \cup_{\alpha \in \Gamma} R_{\alpha}$.

Given the absence of tight pairs we conclude that $x \notin \cup_{\alpha \in C} R_{\alpha}$. To show that $x \notin R_{\gamma}$ for all $\gamma \notin C$ choose an $\alpha \in C$ such that $x_{\alpha}=1$. Then

$$
x_{\alpha}-x_{\gamma}=1 \geq \min \{l(\gamma, \alpha)+\epsilon, 1\}>-l(\alpha, \gamma) .
$$

The last strict inequality follows from the absence of tight pairs.

We now drop the assumption that there are no tight pairs. Again suppose Theorem 2 is false. Amongst all allocation rules $f$ that lead to a counter example choose one where $\Gamma_{f}$ has as few vertices as possible. Notice that $\Gamma_{f}$ must contain a tight pair, $\{\alpha, \theta\}$, say, otherwise the previous argument 
applies. Let $A=\{\beta \in \Gamma:\{\alpha, \beta\}$ is tight $\}$. We construct a new allocation rule $g$ as follows:

1. $g(t)=f(t)$ when $t \notin R_{\alpha} \cup\left\{\cup_{\beta \in A} R_{\beta}\right\}$

2. $g(t)=\alpha$ when $t \in R_{\alpha} \cup\left\{\cup_{\beta \in A} R_{\beta}\right\}$

The allocation rule $g$ will have three properties.

1. $\Gamma_{g}$ has fewer vertices than $\Gamma_{f}$.

2. All 2-cycles in $\Gamma_{g}$ are non-negative.

3. $\Gamma_{g}$ has a negative length cycle.

Such a $g$ gives the lie to the existence of the minimal counterexample $f$ and completes the proof.

The first property of $\Gamma_{g}$ is clearly true. To prove the second property, denote by $l^{g}$ the length function of edges in the network $\Gamma_{g}$.

Observe first that $l^{g}(\gamma, \theta)=l(\gamma, \theta)$ for all $\theta \notin A \cup\{\alpha\}$. It suffices then to verify that $l^{g}(\gamma, \alpha) \geq-l^{g}(\alpha, \gamma)$ for all $\gamma \notin A \cup\{\alpha\}$. Next

$$
l^{g}(\gamma, \alpha)=\min \left\{l(\gamma, \alpha), \min _{\beta \in A}\left\{\inf _{t \in R_{\beta}}\left[t_{\alpha}-t_{\gamma}\right]\right\}\right\} .
$$

For any $\beta \in A, l(\alpha, \beta)=-l(\beta, \alpha)=1$, therefore for all $t \in R_{\beta}$ we have

$$
t_{\alpha}-t_{\gamma}=t_{\alpha}-t_{\beta}+t_{\beta}-t_{\gamma}=-1+t_{\beta}-t_{\gamma}
$$

Hence

$$
l^{g}(\gamma, \alpha)=\min \left\{l(\gamma, \alpha), \min _{\beta \in A}\{-1+l(\gamma, \beta)\}\right\} .
$$

If the first term in the minimand is the smaller, then $l^{g}(\gamma, \alpha)=l(\gamma, \alpha) \geq$ $-l(\alpha, \gamma)=-l^{g}(\alpha, \gamma)$. So we may suppose that $l^{g}(\gamma, \alpha)=-1+l(\gamma, \beta)$ for some $\beta \in A$. If $-1+l(\gamma, \beta) \geq-l(\alpha, \gamma)=-l^{g}(\alpha, \gamma)$ the second property is proved. If not

$$
-1+l(\gamma, \beta)+l(\alpha, \gamma)<0
$$

Therefore $\alpha \rightarrow \gamma \rightarrow \beta \rightarrow \alpha$ is a negative length cycle in $\Gamma_{f}$.

Consider the following type in $T$ : 
1. $t_{\alpha}=\epsilon$

2. $t_{\gamma}=\min [\max \{l(\alpha, \gamma)+2 \epsilon, 0\}, 1]$

3. $t_{\beta}=\max \left\{t_{\gamma}+l(\gamma, \beta)+\epsilon, 0\right\}$

4. $t_{\theta}=0$ for all $\theta \notin\{\alpha, \beta, \gamma\}$

It is easy to see that $t_{\theta} \in[0,1]$ for all $\theta \neq \beta$. We delay the treatment of $\theta=\beta$ for a moment. To complete the proof of the second property it suffices to show that $t \notin \cup_{\theta \in \Gamma} R_{\theta}$.

To see that $t \notin R_{\alpha}$ notice that either $t_{\gamma}-t_{\alpha}>l(\alpha, \gamma) \geq-l(\gamma, \alpha)$ or $t_{\gamma}-t_{\alpha}=1-\epsilon>-l(\gamma, \alpha)$ (for $\epsilon$ sufficiently small). The last strict inequality follows from the fact that $\gamma \notin A$. Also $t_{\alpha}-t_{\beta}>-1=-l(\alpha, \beta)$ so $t \notin R_{\beta}$.

For any $\theta \neq \alpha, \beta, \gamma$ we have $l(\alpha, \theta) \geq 0$. This follows from two facts. First, $\inf \left(r_{\alpha} \mid r \in R_{\alpha}\right)=0$ because $l(\beta, \alpha)=-1$. From this we deduce that $l(\theta, \alpha) \leq 0$ for all $\theta$. From the 2-cycle inequality on $\Gamma_{f}$ it follows that $l(\alpha, \theta) \geq-l(\theta, \alpha) \geq 0$. Now $t \notin R_{\theta}$ since $t_{\theta}-t_{\alpha}=-\epsilon<l(\alpha, \theta)$.

It remains to show that $t_{\beta} \leq 1$ and that $t \notin R_{\gamma}$. We distinguish three cases.

First, assume $l(\alpha, \gamma)=1$. Then $t_{\gamma}=1$ and $t_{\beta}=l(\alpha, \gamma)+l((\gamma, \beta)+\epsilon$. Then $t_{\beta} \leq 1$ because of (12). Furthermore $t_{\beta}-t_{\gamma}=l(\gamma, \beta)+\epsilon>-l(\beta, \gamma)$, therefore $t \notin R_{\gamma}$.

Second, assume $0 \leq l(\alpha, \gamma)<1$. In this case $t_{\gamma}=l(\alpha, \gamma)+2 \epsilon$ (for sufficiently small $\epsilon)$, and either $t_{\beta}=0$, or $t_{\beta}=l(\alpha, \gamma)+l(\gamma, \beta)+3 \epsilon$. Again, $t_{\beta} \leq 1$ because of (12). Observe that $t_{\beta}-t_{\gamma}>l(\gamma, \beta)>-l(\beta, \gamma)$, thus $t \notin R_{\gamma}$.

Now observe that $l(\alpha, \gamma)<0$ is not possible, because together with $l(\gamma, \alpha) \leq 0$ it would imply a negative 2 -cycle.

To prove the last property let $C$ be a negative length cycle in $\Gamma_{f}$. If the pair $\{\alpha, \beta\}$ is not on $C$, then $C$ is present in $\Gamma_{g}$. If the edge $(\alpha, \beta)$ is present in $C$, the contracted version of $C$ has a length that is exactly 1 unit smaller. If the edge $(\beta, \alpha)$ is present in $C$, let $\gamma$ be $\beta$ 's immediate predecessor in $C$. Now $l^{g}(\gamma, \alpha) \leq l(\gamma, \beta)+l(\beta, \alpha)$ and again the the contracted version of $C$ is no longer than the original length of $C$. 
Roberts (1979) offers a characterization of dominant strategy mechanisms in terms of what are called affine maximizers. An allocation rule $f$ is said to be an affine maximizer if there exists non-zero $w \in \Re_{+}^{n}$ and $d \in \Re^{|\Gamma|}$ such that

$$
f(\mathbf{t})=\arg \max _{\alpha \in \Gamma}\left[d_{\alpha}+\sum_{i=1}^{n} w_{i} v^{i}\left(\alpha \mid t^{i}\right)\right] .
$$

By mimicking the payment scheme of a Vickrey-Clarke-Groves scheme, it is easy to show that an allocation rule that is an affine maximizer is dominant strategy incentive compatibility. Roberts shows that any allocation rule that satisfies the 2-cycle inequality must be an affine maximizer. Since affine maximizers are dominant strategy incentive compatible this gives another proof of Theorem 2 in the case when the type space is $\Re^{|\Gamma|}$.

In the case when the type space is $\Re_{+}^{|\Gamma|}$, Theorem 2 can be found in Lavi, Mu'alem and Nisan (2003). The case when the type space is $[0,1]^{|\Gamma|}$ is as far as we know new. The proof technique of Lavi, Mu'alem and Nisan does not apply to this case.

\section{Combinatorial Auctions}

\subsection{General valuations}

We have a set of $M$ distinct goods to allocate. The type of an agent is a vector with one component for each subset of $M$ that corresponds to the value assigned to that subset. We can index $\Gamma$ by the subset allocated to our fixed agent. The type $t$ is now a function defined on subsets of $M$. So, $v(\alpha \mid t)=t_{\alpha} .(10)$ reduces to:

$$
x_{\beta}-x_{\alpha} \geq l(\alpha, \beta)+\epsilon \forall(\alpha, \beta) \in E .
$$

The remainder of the argument follows the unrestricted preference case. We summarize the conclusion as follows:

Theorem 3 Suppose the type space $T$ is $\Re^{2^{M}}, \Re_{+}^{2^{M}}$ or $[0,1]^{2^{M}}$. An allocation rule $f$ for combinatorial auctions is dominant strategy incentive compatible iff.

$$
t_{f(t)}-t_{f(s)} \geq-\left[s_{f(s)}-s_{f(t)}\right] \forall s, t \in T \text {. }
$$


When $T$ is $\Re^{2^{M}}$ or $\Re_{+}^{2^{M}}$ this theorem was first proved in Lavi, Mu'alem and Nisan (2003) using different methods. The case when $T=[0,1]^{2^{M}}$ is new. The proof technique of Lavi, Mu'alem and Nisan does not apply to this case.

\subsection{Non-decreasing valuations}

It is common to impose the free-disposal restriction: $t_{\alpha} \leq t_{\beta}$ if $\alpha \subseteq \beta$. Theorem 3 can be extended to this case. A version of this extenson can be found also in Lavi, Mu'alem and Nisan (2003). The technique there does not extend to the case when the type space is $[0,1]^{2^{M}}$.

If $\alpha \subset \beta$ then $l(\alpha, \beta)=\inf _{t \in R_{\beta}}\left[t_{\beta}-t_{\alpha}\right] \geq 0$, and $l(\beta, \alpha)=\inf _{t \in R_{\alpha}}\left[t_{\alpha}-t_{\beta}\right] \leq$ 0 . An edge $(\alpha, \beta)$ with $\alpha \subset \beta$ will be called a forward edge. Forward edges have non-negative length. System (10) reduces to:

$$
x_{\beta}-x_{\alpha} \geq l(\alpha, \beta)+\epsilon \forall(\alpha, \beta) \in E \text {. }
$$

To ensure that the solution to (14) will be a type in our domain we require that $x_{\beta} \geq x_{\alpha}$ whenever $\alpha \subset \beta$. Notice however, these additional constraints are redundant given the constraints in (14) corresponding to the forward edges. A similar observation applies to the order based domains defined in Lavi, Mu'alem and Nisan (2003).

We omit proofs for the claims made in this subsection since we will provide a proof for a domain that is even more restrictive (see below) than those discussed so far. It is easy to adapt the argument from the more restrictive domain to the more relaxed ones discussed thus far.

\section{Diminishing marginal utility}

Here we consider the case when there are $k$ units of a homogenous good to be allocated. The type, $t$, of an agent is a vector in $[0,1]^{k}$ whose $i^{t h}$ component is the marginal value for the $i^{t h}$ unit. Furthermore, we assume diminishing marginal utilities (DMU), i.e., $t_{i} \geq t_{i+1}$. This is a more restrictive domain than that considered in Bikhchandani, Chatterji and Sen (2003). ${ }^{6}$

\footnotetext{
${ }^{6}$ We have recently been informed that Bikhchandani, Chatterji and Sen have extended their argument to the case of diminishing marginal utilities where each $t_{i} \in\left[0, U_{i}\right]$ and the
} 
Each agent is indifferent between all allocations that give him the same quantity of the good. So we can identify each $\alpha \in \Gamma$ with an integer between 0 and $k$. Then: $v(\alpha \mid t)=\sum_{j=1}^{\alpha} t_{j}$. If $\alpha<\beta$ then

$$
l(\alpha, \beta)=\inf _{t \in R_{\beta}}\left[\sum_{j=1}^{\beta} t_{j}-\sum_{j=1}^{\alpha} t_{j}\right]=\inf _{t \in R_{\beta}}\left[\sum_{j=\alpha+1}^{\beta} t_{j}\right] \geq 0,
$$

and

$$
l(\beta, \alpha)=\inf _{t \in R_{\alpha}}\left[\sum_{j=1}^{\alpha} t_{j}-\sum_{j=1}^{\beta} t_{j}\right]=\inf _{t \in R_{\alpha}}\left[-\sum_{j=\alpha+1}^{\beta} t_{j}\right]=-\sup _{t \in R_{\alpha}}\left[\sum_{j=\alpha+1}^{\beta} t_{j}\right] \leq 0 .
$$

Notice that $|l(\alpha, \beta)| \leq|\alpha-\beta|$. Thus, the two-cycle inequality becomes

$$
\inf _{t \in R_{\beta}}\left[\sum_{j=\alpha+1}^{\beta} t_{j}\right] \geq \sup _{t \in R_{\alpha}}\left[\sum_{j=\alpha+1}^{\beta} t_{j}\right] \text { for all } \alpha<\beta, \alpha, \beta \in\{1, \ldots, k\} .
$$

Notice also that, if $\alpha<\alpha^{\prime}<\beta$ then $l(\alpha, \beta) \geq l\left(\alpha^{\prime}, \beta\right)$ and $l(\beta, \alpha) \leq$ $l\left(\alpha^{\prime}, \alpha\right)$.

Our goal is to prove the following:

Theorem 4 An allocation rule $f$ for diminishing marginal valuations is dominant strategy incentive compatible iff.

$$
\sum_{j=f(s)}^{f(t)} t_{j} \geq \sum_{j=f(s)}^{f(t)} s_{j} \forall t, s \in T .
$$

Fix the allocation rule $f$ and denote by $\Gamma_{f}$ the set of vertices of the associated network. In network terms our goal is to prove the following:

Theorem 4'An allocation rule $f$ for diminishing marginal valuations is dominant strategy incentive compatible iff. all 2-cycles in $\Gamma_{f}$ are nonnegative.

$U_{i}$ 's are not all equal. The domain considered here is more restrictive and not subsumed by their result. 
As usual we confine ourselves to the non-trivial direction. We assume that $\Gamma_{f}$ has all non-negative 2-cycles and will prove that all cycles are nonnegative.

An edge $(a, b)$ in $\Gamma_{f}$ is called tight if

1. $a>b$ and $l(a, b)=-(a-b)$, or

2. $a<b$ and $l(a, b)=0$.

If $\Gamma_{f}$ has no tight edges, $l(a, b)>0$ for all edges $(a, b)$ with $a<b$ and $-l(a, b)<a-b$ for all $a>b$. We assume first that the network $\Gamma_{f}$ has no tight edges. Suppose that $\Gamma_{f}$ consists of the vertices $\left\{i_{1}, i_{2}, \ldots, i_{r}\right\}$. We denote in the following the set $\left\{i_{p}, i_{p+1}, \ldots, i_{q}\right\}$ by $\left[i_{p}, i_{q}\right]$.

Lemma 1 If the network $\Gamma_{f}$ has no negative 2-cycle or tight edges, then

$$
l\left(i_{s}, i_{s+1}\right)=-l\left(i_{s+1}, i_{s}\right)
$$

for $s=1, \ldots, r$.

\section{Proof}

Since the network $\Gamma_{f}$ has no negative 2 -cycle, we have that $l\left(i_{s}, i_{s+1}\right) \geq$ $-l\left(i_{s+1}, i_{s}\right)$. For a contradiction suppose that $l\left(i_{s}, i_{s+1}\right)>-l\left(i_{s+1}, i_{s}\right)$ for some index $s \in\{1, \ldots, r\}$. Choose any $x$ such that

$$
l\left(i_{s}, i_{s+1}\right)>x>-l\left(i_{s+1}, i_{s}\right)
$$

Consider the type $t$ obtained by setting $t_{j}=1$ for $j=1, \ldots, i_{s}, t_{j}=\frac{x}{i_{s+1}-i_{s}}$ for $i_{s}+1 \leq j \leq i_{s+1}$ and $t_{j}=0$ for $j=i_{s+1}+1, \ldots, k$.

Since $\Gamma_{f}$ has no tight edges, $-l\left(i_{q+1}, i_{q}\right)<i_{q+1}-i_{q}$ for all $q<s$. Since $\sum_{j=i_{q}+1}^{i_{q+1}} t_{j}=i_{q+1}-i_{q}$ it follows $t \notin \cup_{q=1}^{s-1} R_{i_{q}}$.

Since $l\left(i_{s}, i_{s+1}\right)>x=\sum_{j=i_{s}+1}^{i_{s+1}} t_{j}>-l\left(i_{s+1}, i_{s}\right)$, it follows that $t \notin R_{i_{s}} \cup$ $R_{i_{s+1}}$.

Since $\Gamma_{f}$ has no tight edges, $l_{i_{q}, i_{q+1}}>0=\sum_{j=i_{q}+1}^{i_{q+1}} t_{j}$ for $q=s+1, \ldots, r-1$, hence $t \notin \cup_{q=s+2}^{r} R_{i_{q}}$.

Therefore $t \notin \cup_{j=1}^{r} R_{i_{j}}$, a contradiction. 
Now suppose $\Gamma_{f}$ has no tight edges but does have a negative cycle, $C$, say. Choose a cycle $C$ such that for $i_{p}$ being the smallest node in $C$ and $i_{q+1}$ being the largest, $\left|i_{q+1}-i_{p}\right|$ is minimized.

Since there is no negative 2-cycle, we have $p<q$. By the choice of $C$ every cycle through any proper subset of vertices in $\left[i_{p}, i_{q+1}\right]$ which does not include $i_{p}$ and $i_{q+1}$ has non-negative length. This implies a triangle inequality. Specifically, for any $r, s \in[p, q+1]$ with $|s-r|<|q+1-p|$ and $r<s$ we have

$$
l\left(i_{r}, i_{s}\right) \geq l\left(i_{r}, i_{r+1}\right)+\ldots+l\left(i_{s-1}, i_{s}\right) .
$$

Indeed, we obtain (15) by applying Lemma 2 to

$$
l\left(i_{r}, i_{s}\right)+l\left(i_{s}, i_{s-1}\right)+l\left(i_{s-1}, i_{s-2}\right)+\ldots+l\left(i_{r-1}, i_{r}\right) \geq 0 .
$$

Therefore the negative cycle $C$ must be of the form

$$
i_{p} \longrightarrow i_{q+1} \longrightarrow \ldots \longrightarrow i_{p-1} \longrightarrow i_{p}
$$

or

$$
i_{p} \longrightarrow i_{p+1} \longrightarrow \ldots \longrightarrow i_{q+1} \longrightarrow i_{p}
$$

We will suppose that $C$ is of the form

$$
i_{p} \longrightarrow i_{q+1} \longrightarrow \ldots \longrightarrow i_{p-1} \longrightarrow i_{p}
$$

The other case can be argued similarly. Since $C$ is a negative length cycle

$$
l\left(i_{p}, i_{q+1}\right)+l\left(i_{q+1}, i_{q}\right)+\ldots+l\left(i_{p+1}, i_{p}\right)<0 .
$$

Lemma 2 If $\Gamma_{f}$ has no tight edges and (16) holds then

$$
\frac{l\left(i_{q}, i_{q+1}\right)}{i_{q+1}-i_{q}}<\frac{l\left(i_{p}, i_{p+1}\right)+\ldots+l\left(i_{q-1}, i_{q}\right)}{i_{q}-i_{p}} .
$$

\section{Proof}

If not, then

$$
\frac{l\left(i_{q}, i_{q+1}\right)}{i_{q+1}-i_{q}} \geq \frac{\left.l\left(i_{p}, i_{p+1}\right)+\ldots+l\left(i_{q-1}, i_{q}\right)\right]}{i_{q}-i_{p}} .
$$


From the DMU assumption we get

$$
\begin{aligned}
l\left(i_{p}, i_{q+1}\right) & \geq\left(i_{q+1}-i_{p}\right) \frac{l\left(i_{q}, i_{q+1}\right)}{i_{q+1}-i_{q}} \\
& =\left(i_{q}-i_{p}\right) \frac{l\left(i_{q}, i_{q+1}\right)}{i_{q+1}-i_{q}}+l\left(i_{q}, i_{q+1}\right) \\
& \geq l\left(i_{p}, i_{p+1}\right)+\ldots+l\left(i_{q-1}, i_{q}\right)+\left(i_{q}, i_{q+1}\right)
\end{aligned}
$$

Invoking Lemma 2 we deduce

$$
l\left(i_{p}, i_{q+1}\right) \geq-l\left(i_{p+1}, i_{p}\right)-\ldots-l\left(i_{q}, i_{q-1}\right)-l\left(i_{q+1}, i_{q}\right) .
$$

This contradicts (16).

Lemma 3 If $\Gamma_{f}$ has no tight edges and (16) holds there is a type $t \in T$, such that $t \notin \cup_{j=1}^{r} R_{i_{j}}$.

\section{Proof}

Let

$$
\lambda=\frac{l\left(i_{p}, i_{p+1}\right)+\ldots+l\left(i_{q-1}, i_{q}\right)}{i_{q}-i_{p}} .
$$

By Lemma $3, \lambda>0$. Since $\Gamma_{f}$ has no tight edges, $-l\left(i_{j+1}, i_{j}\right)<i_{j+1}-i_{j}$ for $j=p, \ldots, q-1$. By Lemma 2 , we get $\lambda<1$.

Consider type $t$ defined as follows:

1. $t_{j}=1$ for $j=1,2, \ldots, i_{p}$,

2. $t_{j}=\lambda-\epsilon$, for $j=i_{p}+1, \ldots, i_{q}$,

3. $t_{j}=\max \left(0, \frac{l\left(i_{q}, i_{q+1}\right)}{i_{q+1}-i_{q}}-\epsilon\right)$ for $j=i_{q}+1, \ldots, i_{q+1}$,

4. $t_{j}=0$ for $j \geq i_{q+1}+1$.

By construction (for $\epsilon$ sufficiently small) and by Lemma $2, t_{i+1} \leq t_{i}$ for $i=1, \ldots, k-1$. Thus $t$ is in our domain $T$. 
The absence of tight edges implies that $t \notin \cup_{j=1}^{p-1} R_{i_{j}}$ and $t \notin \cup_{j=q+2}^{r} R_{i_{j}}$.

If $t_{j}=0$ for $j=i_{q}+1, \ldots, i_{q+1}$, than $t \notin R_{i_{q+1}}$, again due to the absence of tight edges. Otherwise, $\sum_{k=i_{q}+1}^{i_{q+1}} t_{j}=-l\left(i_{q}, i_{q+1}\right)-\left(i_{q+1}-i_{q}\right) * \epsilon<l\left(i_{q}, i_{q+1}\right)$. Hence also in this case $t \notin R_{i_{q+1}}$.

Notice that by definition of $\lambda$ and by Lemma 1 we have

$$
\left(i_{q}-i_{p}\right) \lambda=l\left(i_{p}, i_{p+1}\right)+\ldots+l\left(i_{q-1}, i_{q}\right)=-l\left(i_{p+1}, i_{p}\right)-\ldots-l\left(i_{q}, i_{q-1}\right) .
$$

If $t \in R_{i_{q}}$, we would have

$$
l\left(i_{p}, i_{q}\right) \leq\left(i_{q}-i_{p}\right) *(\lambda-\epsilon)=-l\left(i_{p+1}, i_{p}\right)-\ldots-l\left(i_{q}, i_{q-1}\right)-\left(i_{q}-i_{p}\right) * \epsilon .
$$

which contradicts the choice of the negative cycle. Hence, $t \notin R_{i_{q}}$.

Observe that $t \in R_{i_{p}}$ would imply

$$
l\left(i_{p}, i_{q+1}\right) \geq-l\left(i_{q+1}, i_{p}\right) \geq\left(i_{q}-i_{p}\right) \lambda+l\left(i_{q}, i_{q+1}\right)-\left(i_{q+1}-i_{p}\right) * \epsilon .
$$

From this we would get

$$
l\left(i_{p}, i_{q+1}\right)+l\left(i_{q+1}, i_{q}\right)+\ldots+l\left(i_{p+1}, i_{p}\right) \geq-\left(i_{q+1}-i_{p}\right) * \epsilon .
$$

For $\epsilon$ small enough, this contradicts the choice of $C$.

From what we have seen so far, we know that, for $\epsilon_{0}$ small enough, for all $\epsilon<\epsilon_{0}$ it must hold $t \in \cup_{j=p+1}^{q-1} R_{i_{j}}$. Therefore there exists an $n \in\{p+$ $1, \ldots, q-1\}$ such that $t \in R_{i_{n}}$ for arbitrary small $\epsilon$, where $t$ is defined by this $\epsilon .^{7}$ In the following we consider such $t^{\prime} s$.

Observe that

$$
l\left(i_{p}, i_{n}\right) \leq\left(i_{n}-i_{p}\right) *(\lambda-\epsilon) .
$$

Since $\epsilon$ can be chosen arbitrarily small, we get

$$
l\left(i_{n-j}, i_{n}\right) \leq \lambda\left(i_{n}-i_{n-j}\right)
$$

\footnotetext{
${ }^{7}$ Formally, construct a sequence $\left(\epsilon_{i}\right)_{i=1}^{\infty}$ that converges to 0 . Let $t_{i}$ be the $t$ defined by $\epsilon_{i}$. There must be one $n$ such that $R_{i_{n}}$ contains infinitely many $t^{i}$.
} 
By using the triangle inequality

$$
l\left(i_{p}, i_{n}\right) \geq l\left(i_{p}, i_{p+1}\right)+\ldots+l\left(i_{n-1}, l_{n}\right)
$$

we get

$$
\frac{l\left(i_{p}, i_{p+1}\right)+\ldots+l\left(i_{n-1}, i_{n}\right)}{i_{n}-i_{p}} \leq \frac{l\left(i_{p}, i_{p+1}\right)+\ldots+l\left(i_{q-1}, i_{q}\right)}{i_{q}-i_{p}}
$$

or, equivalently,

$$
\left(i_{q}-i_{p}\right)\left(l\left(i_{p}, i_{p+1}\right)+\ldots+l\left(i_{n-1}, i_{n}\right)\right) \leq\left(i_{n}-i_{p}\right)\left(l\left(i_{p}, i_{p+1}\right)+\ldots+l\left(i_{q-1}, i_{q}\right)\right) .
$$

As some terms cancel, rearranging yields:

$$
\frac{i_{n}-i_{p}}{i_{q}-i_{n}}\left(l\left(i_{n}, i_{n+1}\right)+\ldots+l\left(i_{q-1}, i_{q}\right)\right) \geq l\left(i_{p}, i_{p+1}\right)+\ldots+l\left(i_{n-1}, i_{n}\right) .
$$

Consider any $t^{*} \in R_{q+1}$. Then,

$$
t_{i_{p}+1}^{*}+\ldots+t_{i_{q+1}}^{*}=\left(t_{i_{p}+1}^{*}+\ldots+t_{i_{n}}^{*}\right)+\left(t_{i_{n}+1}^{*}+\ldots+t_{i_{q+1}}^{*}\right) .
$$

From the triangle inequality we get

$$
t_{i_{n}+1}^{*}+\ldots+t_{i_{q+1}}^{*} \geq l\left(i_{n}, i_{q+1}\right) \geq l\left(i_{n}, i_{n+1}\right)+\ldots+l\left(i_{q}, i_{q+1}\right) .
$$

From the diminishing marginal utility condition we get

$$
t_{i_{p}+1}^{*}+\ldots+t_{i_{n}}^{*} \geq\left(i_{n}-i_{p}\right) t_{i_{n}}^{*} \geq \frac{\left(i_{n}-i_{p}\right)}{\left(i_{q}-i_{n}\right)}\left(t_{i_{n}+1}^{*}+\ldots+t_{i_{q}}^{*}\right) .
$$

Further from (17) we deduce that

$$
\begin{aligned}
t_{i_{p}+1}^{*}+\ldots+t_{i_{n}}^{*} & \geq \frac{\left(i_{n}-i_{p}\right)}{\left(i_{q}-i_{n}\right)}\left[t_{i_{n}+1}^{*}+\ldots+t_{i_{q}}^{*}\right] \geq \frac{\left(i_{n}-i_{p}\right)}{\left(i_{q}-i_{n}\right)} l\left(i_{n}, i_{q}\right) \\
& \geq \frac{\left(i_{n}-i_{p}\right)}{\left(i_{q}-i_{n}\right)}\left(l\left(i_{n}, i_{n+1}\right)+\ldots+l\left(i_{q-1}, i_{q}\right)\right) \\
& \geq l\left(i_{p}, i_{p+1}\right)+\ldots+l\left(i_{n-1}, i_{n}\right) .
\end{aligned}
$$

Therefore

$t_{i_{p}+1}^{*}+\ldots+t_{i_{q+1}}^{*} \geq l\left(i_{p}, i_{p+1}\right)+\ldots+l\left(i_{n-1}, i_{n}\right)+l\left(i_{n}, i_{n+1}\right)+\ldots+l\left(i_{q}, i_{q+1}\right)$ 
for all $t^{*} \in R_{q+1}$. Since $l\left(i_{p}, i_{q+1}\right)=\inf _{t^{*} \in R_{i_{q+1}}}\left[t_{i_{p}+1}^{*}+\ldots+t_{i_{q+1}}^{*}\right]$, and from Lemma 1, it follows that

$$
l\left(i_{p}, i_{q+1}\right) \geq-l\left(i_{q+1}, i_{q}\right)-\ldots-l\left(i_{p+1}, i_{p}\right)
$$

contradicting the negative cycle assumption.

Hence $t \notin \cup_{j=1}^{r} R_{i_{j}}$.

Suppose for a contradiction that Theorem 4 is false. Amongst all allocation rules $f$ that lead to a counter example choose one where $\Gamma_{f}$ has as few vertices as possible. Notice that $\Gamma_{f}$ must contain a tight edge, $(a, b)$, say. Since $(a, b)$ is tight, for any $u \in \Gamma_{f}$ with $a<u<b$ either the edge $(a, u)$ or $(u, b)$ will be tight. To see why suppose that $l(a, b)=-l(b, a)=0$. By the DMU assumption, $l(u, b)=0$. By the 2-cycle inequality, $0=l(u, b) \geq$ $-l(b, u)=0$ therefore $-l(b, u)=0$. If $l(a, b)=-l(b, a)=b-a$. By DMU $-l(u, a)=u-a$. By the 2-cycle condition $u-a \geq l(a, u) \geq-l(u, a)=u-a$. Therefore $l(a, u)=-l(u, a)$. Hence, if $\Gamma_{f}$ has a tight edge, it has one of the form $\left(i_{s}, i_{s+1}\right)$ for some $s$.

We will derive from $f$ another allocation rule $g$ by a contraction operation to be described below. If $l\left(i_{s}, i_{s+1}\right)$ is tight and such that $l\left(i_{s}, i_{s+1}\right)=$ $i_{s+1}-i_{s}$. We construct $g$ as follows:

1. $g(t)=f(t)$ if $t \in R_{i_{j}}, j \neq s, s+1$

2. $g(t)=i_{s}$ if $t \in R_{i_{s}} \cup R_{i_{s+1}}$

If $l\left(i_{s}, i_{s+1}\right)=0$ we construct $g$ as follows:

1. $g(t)=f(t)$ if $t \in R_{i_{j}}, j \neq s, s+1$

2. $g(t)=i_{s+1}$ if $t \in R_{i_{s}} \cup R_{i_{s+1}}$

In both cases we say that $g$ was obtained from $f$ by contracting $\left(i_{s}, i_{s+1}\right)$.

We will prove three things about $g$.

1. $\Gamma_{g}$ has one fewer vertex than $\Gamma_{f}$.

2. All 2-cycles in $\Gamma_{g}$ are non-negative. 
3. $\Gamma_{g}$ has a negative length cycle.

Such a $g$ contradicts the existence of the minimal counterexample $f$ and proves the theorem. The first of these claims is clearly true. The following lemma establishes the second claim.

Lemma 4 Suppose $\Gamma_{f}$ has no negative 2-cycles. From amongst all tight edges of the form $\left(i_{s}, i_{s+1}\right)$ choose the one for which $s$ is largest. Let $g$ be obtained from $f$ by contracting the tight edge $\left(i_{s}, i_{s+1}\right)$. Then $\Gamma_{g}$ has no negative 2-cycle.

\section{Proof}

Denote by $l^{g}(a, b)$ the length of the edge $(a, b)$ in $\Gamma_{g}$. Suppose first that $l\left(i_{s}, i_{s+1}\right)=i_{s+1}-i_{s}$. To verify the 2-cycle inequality for $\Gamma_{g}$ it suffices to check that $l^{g}\left(i_{a}, i_{s}\right) \geq-l^{g}\left(i_{s}, i_{a}\right)$ for all $a<s$ and $l^{g}\left(i_{s}, i_{b}\right) \geq-l^{g}\left(i_{b}, i_{s}\right)$ for all $b>s+1$. This is because for any pair $a$ and $b$ that are not part of any tight edge, $l^{g}(a, b)=l(a, b)$.

For $a<s$

$$
l^{g}\left(i_{a}, i_{s}\right)=\min \left\{l\left(i_{a}, i_{s}\right), \inf _{t \in R_{i_{s+1}}} \sum_{j=i_{a}+1}^{i_{s}} t_{j}\right\} .
$$

Since $l\left(i_{s}, i_{s+1}\right)=i_{s+1}-i_{s}$ it follows by DMU that

$$
\inf _{t \in R_{i_{s+1}}} \sum_{j=i_{a}+1}^{i_{s}} t_{j}=i_{s}-i_{a}
$$

Therefore $l^{g}\left(i_{a}, i_{s}\right)=l\left(i_{a}, i_{s}\right) \geq-l\left(i_{s}, i_{a}\right)=l^{g}\left(i_{s}, i_{a}\right)$.

For $b>s+1$

$$
-l^{g}\left(i_{b}, i_{s}\right)=\max \left\{-l\left(i_{b}, i_{s}\right), \sup _{t \in R_{i_{s+1}}} \sum_{j=i_{s}+1}^{i_{b}} t_{j}\right\} .
$$

If $-l\left(i_{b}, i_{s}\right)$ is the larger of the two terms in the maximand, then $-l^{g}\left(i_{b}, i_{s}\right)=$ $-l\left(i_{b}, i_{s}\right) \leq l\left(i_{s}, i_{b}\right)=l^{g}\left(i_{s}, i_{b}\right)$. So, we suppose that

$$
-l^{g}\left(i_{b}, i_{s}\right)=\sup _{t \in R_{i_{s+1}}} \sum_{j=i_{s}+1}^{i_{b}} t_{j} .
$$


If $-l^{g}\left(i_{b}, i_{s}\right) \leq l\left(i_{s}, i_{b}\right)=l^{g}\left(i_{s}, i_{b}\right)$ we are done. If not

$l\left(i_{s}, i_{b}\right)=l^{g}\left(i_{s}, i_{b}\right)<\sup _{t \in R_{i_{s+1}}} \sum_{j=i_{s}+1}^{i_{b}} t_{j} \leq\left(i_{s+1}-i_{s}\right)-l\left(i_{b}, i_{s+1}\right)=-l\left(i_{s+1}, i_{s}\right)-l\left(i_{b}, i_{s+1}\right)$.

Hence

$$
l\left(i_{s}, i_{b}\right)+l\left(i_{b}, i_{s+1}\right)+l\left(i_{s+1}, i_{s}\right)<0 .
$$

In words $i_{s} \rightarrow i_{b} \rightarrow i_{s+1} \rightarrow i_{s}$ is a negative cycle. We will use the cycle to derive a type that is not in $\cup_{j=1}^{r} R_{i_{j}}$. Consider now the following system:

$$
\begin{gathered}
X+Y \geq l\left(i_{s}, i_{b}\right)+\epsilon \\
X \leq-l\left(i_{s+1}, i_{s}\right)-\epsilon \\
Y \leq-l\left(i_{b}, i_{s+1}\right)-\epsilon \\
1 \geq \frac{X}{i_{s+1}-i_{s}} \geq \frac{Y}{i_{b}-i_{s+1}} \geq 0
\end{gathered}
$$

We show that this system is feasible by showing that the following program has optimal objective function value of zero.

$$
\min A+B
$$

subject to

$$
\begin{gathered}
X+Y \geq l\left(i_{s}, i_{b}\right)+\epsilon \\
X-A \leq-l\left(i_{s+1}, i_{s}\right)-\epsilon \\
Y-B \leq-l\left(i_{b}, i_{s+1}\right)-\epsilon \\
1 \geq \frac{X}{i_{s+1}-i_{s}} \geq \frac{Y}{i_{b}-i_{s+1}} \geq 0 \\
A, B \geq 0
\end{gathered}
$$

To see that this program is feasible set $X=i_{s+1}-i_{s}$ and $Y=i_{b}-i_{s+1}$.

Suppose, for a contradiction, that at optimality $A+B>0$. It is easy to see that at optimality the first three constraints must bind. So

$$
l\left(i_{s}, i_{b}\right)+\epsilon=-l\left(i_{s+1}, i_{s}\right)-l\left(i_{b}, i_{s+1}\right)-2 \epsilon+A+B .
$$


Therefore

$$
l\left(i_{s}, i_{b}\right)+l\left(i_{s+1}, i_{s}\right)+l\left(i_{b}, i_{s+1}\right)=-3 \epsilon+A+B>0
$$

for $\epsilon$ sufficiently small, contradicting (18).

Consider now the following type $t$ :

1. $t_{j}=1$ for $j=1, \ldots, i_{s}$

2. $t_{j}=\frac{X}{i_{s+1}-i_{s}}$ for $j=i_{s}+1, \ldots, i_{s+1}$

3. $t_{j}=\frac{Y}{i_{b}-i_{s+1}}$ for $j=i_{s+1}+1, \ldots, i_{b}$

4. $t_{j}=0$ for $j=i_{b}+1, \ldots, k$

We show that $t \notin \cup_{j=1}^{r} R_{i_{j}}$

1. $t \notin R_{i_{s}}$

This follows from the fact $\sum_{j=i_{s}+1}^{i_{b}} t_{j}>l\left(i_{s}, i_{b}\right) \geq-l\left(i_{b}, i_{s}\right)$.

2. $t \notin R_{i_{s+1}}$

This follows from the fact that $\sum_{j=i_{s}+1}^{i_{s+1}} t_{j}<-l\left(i_{s+1}, i_{s}\right) \leq l\left(i_{s}, i_{s+1}\right)$.

3. $t \notin R_{i_{b}}$

This follows from the fact that $\sum_{j=i_{s+1}+1}^{i_{b}} t_{j}<-l\left(i_{b}, i_{s+1}\right) \leq l\left(i_{s+1}, i_{b}\right)$.

4. $t \notin \cup_{j=b+1}^{r} R_{i_{j}}$

If $t \in R_{i_{q}}$ for some $q>b$ then $l\left(i_{b}, i_{q}\right)=0$ implying by DMU that $l\left(i_{q-1}, i_{q}\right)=0$. By the 2-cycle inequality it follows that $-l\left(i_{q}, i_{q+1}\right)$ contradicting the choice of $\left(i_{s}, i_{s+1}\right)$.

5. $t \notin \cup_{j=1}^{s-1} R_{i_{j}}$

If $t \in R_{i_{q}}$ for some $q<s$ then $l\left(i_{q}, i_{b}\right) \geq-l\left(i_{b}, i_{q}\right) \geq i_{s}-i_{q}+X+Y$. However for any $\delta>0$ sufficiently small, there is a type $t^{\prime} \in R_{i_{b}}$ such that $\sum_{j=i_{s}+1}^{i_{b}} t_{j}^{\prime}=l\left(i_{s}, i_{b}\right)+\delta$. Thus

$$
l\left(i_{q}, i_{b}\right) \leq i_{s}-q+l\left(i_{s}, i_{b}\right)+\delta<i_{s}-i_{q}+X+Y \leq l\left(i_{q}, i_{b}\right)
$$

a contradiction. 
6. $t \notin \cup_{j=s+2}^{b-1} R_{i_{j}}$

If $t \in R_{i_{q}}$ where $i_{s+1}<i_{q}<i_{b}$ then $-l\left(i_{q}, i_{s+1}\right) \leq l\left(i_{s+1}, i_{q}\right) \leq \frac{\left(i_{q}-i_{s+1}\right) Y}{i_{b}-i_{s+1}}$.

However, by the DMU condition

$$
-l\left(i_{q}, i_{s+1}\right) \geq-\frac{\left(i_{q}-i_{s+1}\right) l\left(i_{b}, i_{s+1}\right)}{i_{b}-i_{s+1}} .
$$

Therefore

$$
\frac{\left(i_{q}-i_{s+1}\right) Y}{i_{b}-i_{s+1}} \geq-\frac{\left(i_{q}-i_{s+1}\right) l\left(i_{b}, i_{s+1}\right)}{i_{b}-i_{s+1}} .
$$

In other words $Y \geq-l\left(i_{b}, i_{s+1}\right)$ which contradicts the choice of $Y$.

The case when $l\left(i_{s}, i_{s+1}\right)=0$ follows similarly.

Observe that the length of an edge can only decrease after a contraction operation. For example, if $a<b<c$ and $(a, b)$ is a tight pair with $l(b, a)=$ $-(a-b)$ then

$$
l^{g}(c, a) \leq l(c, b)+l(b, a) .
$$

Hence if $\Gamma_{f}$ has a negative cycle, $\Gamma_{g}$ must have it as well. This observation completes the proof of the third claim about $g$.

\section{Acknowledgement}

The research of the third author was supported in part by NSF grant ITR IIS-0121678. A portion of this research was performed at the Universiteit Maastricht under the auspices of a grant of the Dutch Science Foundation (NWO) and METEOR (Maastricht Research School of Economics, Technology and Organizations). We thank Ron Lavi and Noam Nisan for useful comments.

\section{References}

1. Bikhchandani, S., S. Chatterji and A. Sen. 'Incentive Compatibility in Multi-unit Auctions', manuscript, 2003. 
2. Jehiel, P. and B. Moldovanu, 'Efficient Design with Interdependent Valuations', Econometrica, 69, 1237-1259, 2001.

3. Krishna, V. and M. Perry, 'Efficient Mechanism Design', manuscript, 1997.

4. Lavi, R., A. Mu'alem and N. Nisan. 'Towards a Characterization of Truthful Combinatorial Auctions', manuscript, 2003.

5. Myerson, R. 'Optimal Auction Design', Mathematics of Operations Research, 6, 58-73, 1981.

6. Roberts, K. 'The Characterization of Implementable Choice Rules', in Aggregation and Revelation of Preferences, J. J. Laffont (ed.), North Holland Publishing Company, 1979.

7. Rochet, J.-C. 'A condition for rationalizability in a quasi-linear context', Journal of Mathematical Economics, 16, 191-200, 1987.

8. Rozenshtrum, I. "Dominant Strategy Implementation with Quasi-linear Preferences", manuscript, 1999. 